\title{
Die Bedeutung von Ärztenetzwerken
}

In den letzten Jahren hat die Bedeutung von Ärztenetzwerken, ob mit oder ohne Budgetverantwortung, zugenommen. Aus der ursprünglich losen Zusammenarbeit mit häufigen Doppelspurigkeiten ist an vielen Orten eine koordinierte, konzentrierte und kompetente Kooperation entstanden. Dank vermehrter Treffen der Beteiligten und gemeinsamer Erstellung von Behandlungsabläufen konnten Doppelspurigkeiten auf ein Minimum reduziert werden.

Die meisten Ärztenetzwerke umfassen eine mehr oder weniger grosse Region und gruppieren sich um eine oder mehrere Kliniken. Sie sind in der Regel organisch gewachsen und verknüpfen im Idealfall Haus-, Beleg-, Konsiliar-, Spezial- und angestellte Klinikärzte zu einer Behandlungskette. Es ist wichtig, dass in dieser Behandlungskette jede Ärztegruppe die Aufgaben übernimmt, zu der sie am besten befähigt ist, womit die Bedeutung jeder einzelnen Ärztegruppe nicht nur anerkannt und wahrgenommen wird, sondern die Beteiligten sich gegenseitig unterstützen und die Behandlungskette für den Patienten optimiert werden kann. Solche regionalen Treffen können beispielsweise über die Bezirksärztegesellschaften organisiert werden und nach Bedarf 1-2× jährlich stattfinden. Die verbesserte Zusammenarbeit der zuweisenden Ärzte mit den Klinikärzten hat zu einer Erhöhung der Patientensicherheit und zu einem rascheren Ablauf bei den administrativen Vorgaben geführt.

\section{Grundpfeiler der medizinischen Versorgung}

In vielen Regionen der Schweiz ist das Ärztenetzwerk zum Grundpfeiler der medizinischen Versorgung geworden. Für die Patientenbetreuung bedeutet es eine verbesserte medizinische Behandlung, da die Spezialärzte jederzeit beigezogen werden können und damit ihre Kompetenz und ihr entsprechendes Know-how ständig verfügbar wird. Die Medizin hat sich in den letzten Jahren immer stärker spezialisiert, so dass immer mehr Spezialisten mit unterschiedlicher Fachkenntnis in einem Netzwerk eingebunden werden müssen. Es versteht sich von selbst, dass diese Spezialärzte nicht alle Angestellte der Kliniken sein können, da der notwendige «case load» innerhalb einer Klinik nicht unbedingt vorhanden ist.

\section{Die Spitalleitungen müssen Ärztenetzwerke in ihrer Strategieplanung berücksichtigen}

Spitalmanager und Politiker müssen erkennen, dass Ärztenetzwerke Grundlage einer optimalen medizinischen Betreuung bei einzelnen Krankheiten und/oder einer geo- graphischen Region sind und deshalb in ihrer organisch gewachsenen Struktur zu unterstützen sind.

Die Zunahme der Bürokratie in den Kliniken im Rahmen des New Public Management hat an vielen Kliniken zu Veränderungen der Organisationsstrukturen geführt. Daraus abgeleitet ist auch der Wunsch, vermehrt klinikeigene Ärzte anzustellen. Diese sind in der Regel günstiger als Belegärzte und können durch die Einbindung in den Klinikalltag auch besser geführt werden. Es besteht aber die Gefahr, dass Klinikärzte, sobald sie optimal ausgebildet sind, abspringen, um als freipraktizierende Spezialärzte weiterzuarbeiten. Es ist jedoch wichtig, dass auch gutausgebildete Kolleginnen und Kollegen als Angestellte der Klinik erhalten bleiben. Dies bedingt aber, dass Anstrengungen unternommen werden, um die Attraktivität des Arbeitsplatzes im Spital auch für diese Ärzte zu erhalten - nicht zuletzt, weil es heutzutage eine grosse Portion Idealismus braucht, um als Chefärztin zu arbeiten. Dies, weil unter anderem die Komplexität der Aufgaben stark zugenommen hat und vor allem die Aufwände zur Erledigung der bürokratischen Vorgaben teilweise unerträglich gestiegen sind und weiter steigen. Auch muss immer mehr nichtmedizinisches Personal mitfinanziert werden, obwohl die Einnahmemöglichkeiten in der Regel gleichgeblieben sind. Um zu vermeiden, dass gutausgebildete Fachärzte abspringen, müssen sie die Möglichkeit haben, aktiv an der Gestaltung des «Gebildes Spital» mitarbeiten zu können, d.h., sie müssen in die Führungsstrukturen der Kliniken eingebunden sein.

\section{Zukunftsperspektiven}

Die verbesserte Zusammenarbeit der Klinikärzte mit den freipraktizierenden Ärzten ermöglicht auch eine sinnvollere, effizientere Organisation der Notfalldienste: Das gemeinsame Anbieten einer flächendeckenden Notfallversorgung, wie das schon in einigen Regionen der Schweiz realisiert ist, zeigt eine erfreuliche Zukunftsperspektive, die vor allem auch die Grundversorger entlasten und zur Attraktivität des Berufes des Hausarztes beitragen wird. All diese Gründe zeigen, wie wichtig der Erhalt und die Pflege des gut funktionierenden Ärztenetzwerkes für eine sinnvolle Gesundheitspolitik sind.

Dr. med. Brigitte Muff, Mitglied des Zentralvorstandes der FMH 